\title{
Estudo macroscópico do aparelho digestório de Gracilinanus microtarsus (Wagner, 1842) (Mammalia: Didelphidae)
}

\author{
Luis Miguel Lobo ${ }^{1^{*}}$ \\ Amilton Cesar dos Santos ${ }^{1}$ \\ Ricardo Alexandre Rosa ${ }^{2}$ \\ Carlos Eduardo Ambrosio 5 \\ Denis Cristiano Briani ${ }^{3}$ \\ Gerlane M. Costa ${ }^{4}$ \\ Ana Flávia Carvalho ${ }^{2}$ \\ Celina Almeida Furlanetto Mançanares ${ }^{2,5}$ \\ ${ }^{1}$ Faculdade de Medicina Veterinária e Zootecnia, Universidade de São Paulo \\ PPG em Anatomia dos Animais Domésticos e Silvestres \\ Avenida Prof. Dr. Orlando Marques de Paiva, CEP 05508-270, São Paulo $\square$ SP, Brasil \\ ${ }^{2}$ Centro Universitário da Fundação de Ensino Octávio Bastos \\ ${ }^{3}$ Faculdades Integradas Claretianas \\ ${ }^{4}$ Universidade do Estado do Mato Grosso \\ ${ }^{5}$ Faculdade de Zootecnia e Engenharia de Alimentos \\ * Autor para correspondência \\ miguel_simons@hotmail.com
}

Submetido em 30/07/2013

Aceito para publicação em 03/12/2013

\section{Resumo}

Gracilinanus microtarsus é um pequeno marsupial pertencente à família Didelphidae. Possui hábito alimentar onívoro/frugívoro sendo, portanto, uma espécie de grande importância ecológica, por ser dispersora de sementes. O objetivo deste estudo é descrever a morfologia macroscópica do aparelho digestório do $G$. microtarsus. Foram utilizados quatro animais fixados em formaldeído $10 \%$. Os órgãos foram dissecados, mensurados e fotografados. Os animais estudados apresentaram a fórmula dentária 2x I 5/4 C1/1 P 3/3 M 4/4. Esta é a fórmula dentária de toda a família Didelphidae. O dorso da língua apresentou papilas valadas, fungiformes e filiformes. O esôfago tubular revelou as porções cervical, torácica e abdominal. O estômago monocavitário era composto por região glandular e aglandular e pregas gástricas. $\mathrm{O}$ intestino delgado apresentou três porções: duodeno, jejuno e íleo. O intestino grosso era composto por: ceco, cólon e reto. A glândula salivar parótida era a maior e apresentava formato achatado. A glândula salivar sublingual, que era a menor, apresentava formato achatado e alongado. A glândula salivar mandibular apresentava formato ovalado. O pâncreas apresentou forma dispersa e aspecto lobulado. O fígado apresentava formato de cúpula e era composto pelos lobos medial direito, quadrado, lateral direito, medial esquerdo, lateral esquerdo e caudado. $\mathrm{O}$ aparelho digestório dos animais estudados é semelhante ao de marsupiais descritos na literatura.

Palavras-chave: Aparelho digestório; Guaiquica; Macroscopia; Marsupiais 


\section{Abstract}

Macroscopic study of the digestive tract of Gracilinanus microtarsus (Wagner, 1842) (Mammalia: Didelphidae). Gracilinanus microtarsus is a small marsupial species belonging to the Didelphidae family. It has an omnivorous/frugivorous feeding habit and, therefore, it has a great ecological importance, because it is a seed-dispersing species. This article aims to describe the macroscopic morphology of the digestive tract in G. microtarsus. We used 4 animals fixed in $10 \%$ formaldehyde. The organs were dissected, measured, and photographed. The animals under study had the dental formula 2x I 5/4 C 1/1 P 3/3 M 4/4. This is the dental formula of the whole Didelphidae family. The dorsum of the tongue had vallate, fungiform, and filiform papillae. Tubular esophagus evidenced the cervical, thoracic, and abdominal portions. The unicavitary stomach consisted of glandular and aglandular region and gastric folds. Small intestine had 3 portions: duodenum, jejunum, and ileum. Large intestine consisted of: cecum, colon, and rectum. Parotid salivary gland was the largest and it had a flattened shape. The sublingual salivary gland, which was the smallest, had a flattened and elongated shape. Mandibular salivary gland had an oval shape. Pancreas had a dispersed shape and lobulated aspect. Liver had a dome shape and it consisted of the lobes right medial, square, right side, left medial, left side, and caudate. The digestive tract of the animals under study is similar to the marsupial species described in the literature.

Key words: Digestive tract; Guaiquica; Macroscopy; Marsupials

\section{Introdução}

Gracilinanus microtarsus é um pequeno marsupial de hábito noturno e arborícola com massa corporal entre 20 e 44 g (MARTINS; ARAÚJO, 2008), pertencente à família Didelphidae (ORR, 1986; EISENBERG; REDFORD, 1999). Esta espécie é conhecida popularmente no Brasil como guaiquica, guaiquiquinha e catita (GONÇALVES et al., 2009).

No Brasil a distribuição geográfica desta espécie abrange principalmente a Mata Atlântica do sul e sudeste brasileiro, do Estado de Minas Gerais ao Rio Grande do Sul (HERSHKOVITZ, 1992; COSTA et al., 2003).

Possui hábito alimentar onívoro-frugívoro se alimentando de insetos, frutos e pequenos vertebrados, sendo, portanto uma espécie de grande importância ecológica por ser dispersora de sementes de algumas árvores. Sendo esta uma espécie capaz de percorrer grandes distâncias no interior das matas a procura de alimento acaba espalhando junto com suas fezes as sementes dos frutos ingeridos (FONTES et al., 2007).

A morfofisiologia dos animais é o resultado da seleção natural que favorece a aquisição efetiva de energia dos alimentos, e evita que se tornem alimentos de outros animais. A força seletiva faz com que os animais adquiram adaptações para garantir sua sobrevivência, e em decorrência dessas variações adaptativas, vários métodos alimentares são encontrados no reino animal (RANDALL et al., 2000).

Para tanto, os mamíferos apresentam um conjunto de órgãos formando o aparelho digestório, o qual compreende os órgãos relacionados com a apreensão e recepção do alimento, redução mecânica, digestão química, absorção de nutrientes e líquidos e a eliminação de resíduos não absorvidos. $\mathrm{O}$ aparelho digestório é formado pela boca e órgãos da cavidade oral, faringe, esôfago, estômago, intestino delgado e intestino grosso (DYCE et al., 2010).

Devido à importância ecológica do G. microtarsus, objetivou-se caracterizar morfologicamente os órgãos do aparelho digestório da espécie, relacionando com seus hábitos alimentares, fornecendo assim, dados que possam auxiliar futuros programas de conservação e criação em cativeiro, além de estudos relacionados à seleção natural, adaptações anatômicas e fisiológicas dos mamíferos em geral.

\section{Material e Métodos}

\section{Animais}

Foram utilizados quatro Gracilinanus microtarsus, adultos, dois machos e duas fêmeas, provenientes do Município de Alta Floresta MT (Latitude: 954’0”Sul 
e Longitude: 5554'0"Oeste) (Cidade-Brasil, 2013) os quais foram doados ao Laboratório de Ciências Morfológicas do Centro Universitário da Fundação de Ensino Octávio Bastos (IBAMA 02001.007176/03-69). Estes animais foram utilizados em pesquisas anteriores e por esta razão já se encontravam fixados em formaldeído a $10 \%$.

\section{Análise macroscópica e morfométrica}

Para análise macroscópica do aparelho digestório de guaiquica, foi realizada uma incisão da pele, na linha mediana ventral, os órgãos componentes do canal alimentar e suas glândulas anexas foram identificados, mensurados e descritos in situ" e "ex situ" e a documentação fotográfica macroscópica foi realizada por meio de uma câmara digital Sony Mavica 3.2 MP.

Os órgãos do aparelho digestório foram mensurados com paquímetro de precisão Mitotuyo ${ }^{\circledR}$ e os valores da média \pm DP (desvio padrão) dispostos em tabelas.

A nomenclatura macroscópica utilizada foi referida conforme International Commitee on Veterinary Gross Anatomical Nomenclature (2012).

\section{Resultados}

\section{Análise macroscópica}

A cavidade oral da guaiquica começa entre os lábios e termina na faringe, sendo dividida pelos dentes, lábios e bochechas em um vestíbulo (Figura 1A). A cavidade oral propriamente dita estava localizada no interior das arcadas dentarias, sendo coberta pelo palato, e limitada lateralmente pelos dentes e gengivas, e assoalhada pela língua (Figura 1A). Os lábios mostraram-se delgados (Figura 1B).

O guaiquica possui dentes braquidontes. Apresentaram dez dentes incisivos superiores (Figura $1 \mathrm{C} \mathrm{e} 1 \mathrm{D}$ ) e oito dentes incisivos inferiores (Figura 1E e 1F) (totalizando 18 incisivos), dois caninos superiores (Figura 1C e 1D) e dois caninos inferiores (Figura 1E e 1F) (totalizando quatro caninos), seis pré-molares superiores (Figura 1C e 1D) e seis pré-molares inferiores (Figura 1E e 1D) (totalizando 12 pré-molares), e oito molares superiores (Figura 1C e 1D) e oito molares inferiores (Figura 1E e 1D) (totalizando 16 molares) apresentando a fórmula dentária 2x I 5/4 C1/1 P 3/3 M 4/4 (Figura 2C, 2D, 2E e 2F).

A língua do G. microtarsus estendeu-se da orofaringe, e ocupou a maior parte da cavidade oral. Revelou raiz e corpo fixo e ápice livre, preso ao assoalho apenas pelo frênulo lingual. A língua dessa espécie exibiu papilas filiformes em toda a superfície dorsal, papilas fungiformes marginais localizadas na margem do ápice, três papilas valadas, uma central e duas laterais na região dorsal da raíz e papilas fungiformes distribuídas em toda a superfície dorsal (Figura $1 \mathrm{G}$ ). As medidas da língua estão dispostas detalhadamente na Tabela 1 .

O esôfago apresentou formato tubular, delgado, começando dorsalmente à cartilagem cricoidea da faringe, e seguindo a traqueia ao longo do pescoço, inclinando-se para a esquerda (Figuras 2A e 2B), reassumindo uma forma simétrica acima na região torácica, onde seguiu no mediastino, passando sobre o coração antes de entrar no hiato esofágico do diafragma. Após adentrar a cavidade abdominal, o esôfago uniu-se ao estômago no cárdia (Figura 2C). As medidas do órgão estão dispostas detalhadamente na Tabela 1.

O estômago dos animais estudados era do tipo monocavitário simples, interposto entre o esôfago e duodeno, e sua porção cranial estava em íntimo contato com o músculo diafragma e com o fígado (Figura 2B), dispondo-se caudal a este, se observado pela vista ventral do animal (Figura 2B), apresentando formato peculiar, piriforme (Figura 2C), com extremidade pilórica curta.

Internamente, o estômago do G. microtarsus era composto por uma região aglandular e uma porção glandular na parte mais dilatada do órgão (Figura 2D). O cárdia estava localizado na porção cranial do estômago (Figura 2C e Tabela 1).

Os dados morfométricos do estômago estão dispostos na Tabela 1 .

$\mathrm{O}$ intestino delgado era dividido em três porções, duodeno, seguido do jejuno e íleo, (Figura 2A e 2B). A Tabela 1 apresenta dados de medidas do intestino delgado em suas porções. 
FIGURA 1: Fotomacrografia da cavidade oral de Gracilinanus microtarsus. A: palato duro (Pd), palato mole (Pm), vestíbulo oral (V), arco orofaríngeo (Ao) e língua (L). Escala de barra: $1 \mathrm{~mm}$. B: pregas palatinas (P) e os lábios (L). Escala de barra: $1 \mathrm{~mm}$. C: fissura labial (F), seis dentes pré-molares (P e P1), diastemas entre o canino e o primeiro pré-molar (D) e entre o primeiro e o segundo pré-molar (D1), oito dentes molares (M e M1), na arcada dentária superior. Escala de barra: 1mm. D: fissura labial (F), dez dentes incisivos (I, I1 e I2), diastema entre o primeiro e o segundo incisivo, entre o último incisivo e o canino (D1), dois dentes caninos ( $\mathrm{C}$ e C1), diastemas entre o canino e o primeiro pré-molar (D2) e entre o primeiro e o segundo pré-molar (D3), da arcada dentária superior e papila palatina (seta fina). Escala de barra: 1mm. E: oito dentes molares (M e M1), seis dentes pré-molares (P e P1), diastemas entre o terceiro e o segundo pré-molar, entre o segundo e o primeiro pré-molar e entre o primeiro pré-molar e o canino (D, D1 e D2, respectivamente). Escala de barra: $1 \mathrm{~mm}$. F: dois dentes caninos (C e C1), oito dentes incisivos (I e I1) e diastemas entre o primeiro pré-molar e o canino (D), entre o canino e o último pré-molar (D1) e entre os primeiros incisivos (D2) da arcada dentária inferior. Escala de barra: $1 \mathrm{~mm}$. G: vista dorsal da língua indicando as três porções: ápice (A), corpo (C) e raiz (R). Observar papila valada central $(\mathrm{Vc})$, e papilas valadas laterais $(\mathrm{Vl})$, projeções palilares $(\mathrm{P})$, papila fungiforme $(\mathrm{F})$, papilas filiformes $(\mathrm{Fl})$, papilas fungiformes marginais $(\mathrm{Fm})$, localizadas no ápice da língua. Escala de barra: $1 \mathrm{~mm}$.
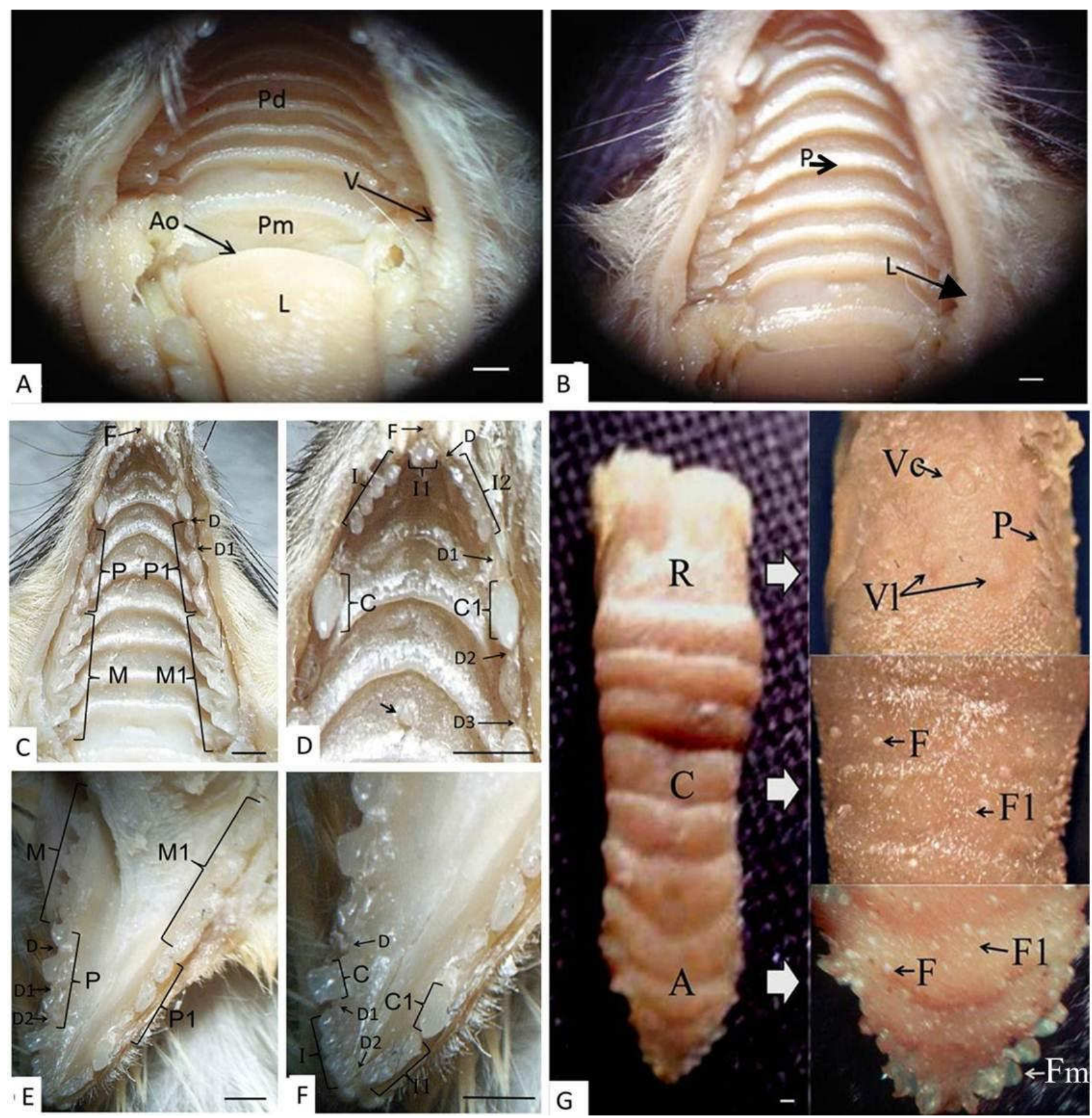

G 
TABELA 1: Biométrica do aparelho digestório dos Gracilinanus microtarsus.

\begin{tabular}{|c|c|c|c|c|}
\hline & & Comprimento cm & Largura cm & Espessura cm \\
\hline \multirow{3}{*}{ Língua } & Raiz & 0,4 & $0,55 \pm 0,06$ & 0,9 \\
\hline & Corpo & $0,45 \pm 0,06$ & $0,48 \pm 0,05$ & $0,38 \pm 0,05$ \\
\hline & Ápice & 0,3 & 0,4 & 0,1 \\
\hline \multirow{4}{*}{ Esôfago } & Porção cervical & $1,2 \pm 0,32$ & 0,2 & - \\
\hline & Porção torácica & $1,08 \pm 1,08$ & 0,2 & - \\
\hline & Porção abdominal & $0,43 \pm 0,05$ & 0,2 & - \\
\hline & & Comprimento cm & \multicolumn{2}{|c|}{ Diâmetro cm } \\
\hline \multirow{3}{*}{ Estômago } & & $1,35 \pm 0,3$ & & \\
\hline & Porção pilórica & & \multicolumn{2}{|c|}{$1,00 \pm 0,08$} \\
\hline & Porção fundica & & \multicolumn{2}{|c|}{$0,68 \pm 0,15$} \\
\hline \multirow{3}{*}{ Intestino delgado } & Duodeno & $1,83 \pm 0,19$ & \multicolumn{2}{|c|}{$0,58 \pm 0,10$} \\
\hline & Jejuno & $15,85 \pm 0,58$ & \multicolumn{2}{|c|}{0,4} \\
\hline & Íleo & $2,00 \pm 0,22$ & \multicolumn{2}{|c|}{$0,33 \pm 0,05$} \\
\hline \multirow{4}{*}{ Intestino grosso } & Ceco & $3,13 \pm 0,0,25$ & \multicolumn{2}{|c|}{$0,40 \pm 0,08$} \\
\hline & Cólon ascendente & $2,15 \pm 0,0,06$ & \multicolumn{2}{|c|}{$0,55 \pm 0,0,13$} \\
\hline & Cólon descendente & $2,08 \pm 0,0,17$ & \multicolumn{2}{|c|}{$0,55 \pm 0,0,06$} \\
\hline & Reto & $3,38 \pm 0,0,45$ & \multicolumn{2}{|c|}{$0,33 \pm 0,05$} \\
\hline
\end{tabular}

$\pm=$ Desvio padrão.

O intestino grosso do G. microtarsus estava dividido em três partes, ceco, cólon e reto (Figura 2A e 2B). O ceco dos animas estudados apresentou-se relativamente curto, voltado em direção ao cólon, e o reto relativamente longo se comparado com o cólon e com o ceco. Os dados morfométricos do ceco colón e reto estão dispostos na Tabela 1 .

$O$ reto estava inserido na cloaca e abriu-se na mesma pelo ânus. A cloaca mostrou-se uma cavidade comum ao aparelho urogenital e digestório (Figura 2A e 2E). A abertura anal localiza-se dorsalmente em relação à abertura genital.

A glândula salivar parótida dos animais estudados, era a maior se comparada às demais, apresentando formato achatado e iniciava-se na base da orelha (Figuras 3A e 3B).

A glândula salivar sublingual, que era a menor comparada às outras, estava inserida na glândula parótida, apresentando formato achatado, alongado (Figuras $3 \mathrm{Ae}$ 3B). Já a glândula salivar mandibular revelou formato ovalado e estava localizada ventralmente à glândula parótida e sublingual (Figuras 3A e 3B).

O pâncreas situou-se no antímero direito, e se estendeu do início do piloro até a porção final do duodeno, abrindo-se no ducto pancreático (Figura 2B). Apresentou forma dispersa, com superfície irregular e aspecto lobulado, com vários vasos sanguíneos (Figura $3 \mathrm{C})$.

O fígado do Gracilinanus microtarsus estava localizado na região abdominal cranial, em íntimo contato com o diafragma (Figuras $2 \mathrm{~A} \mathrm{e} \mathrm{B)}$ ). Apresentou formato de cúpula sendo composto por seis lobos: medial direito e quadrado, onde a vesícula biliar localizavase (na fossa da vesícula biliar), lateral direito, medial esquerdo, lateral esquerdo e lobo caudado (Figuras 3 $\mathrm{D}$ e E). 
FIGURA 2: Fotomacrografia em vista ventral "in situ" dos órgãos na cavidade abdominal e torácica. A: seta vermelha indicando a região onde a figura foi aumentada, traqueia $(\mathrm{T})$, esôfago $(\mathrm{E})$, coração $(\mathrm{C})$, pulmão direito $(\mathrm{P})$, diafragma $(\mathrm{Di})$, fígado $(\mathrm{F})$, duodeno (D), baço (B), íleo (I), mesentério (M), cólon descendente $(\mathrm{Cd})$, jejuno (J), ceco $(\mathrm{Cc})$ e bexiga $(\mathrm{Be})$. Escala de barra: $1 \mathrm{~cm}$. B: fotomacrografia $\square$ ex situ $\square$ em vista dorsal do: esôfago (E), estômago (Es), fígado (F), baço (Ba), duodeno (D), pâncreas (P), jejuno $(\mathrm{J})$, cólon ascendente $(\mathrm{Ca})$, cólon descendente $(\mathrm{Cd})$, reto $(\mathrm{R})$ e cloaca $(\mathrm{C})$. Escala de barra: $1 \mathrm{~cm}$. C: vista ventrolateral do estômago mostrando porção final do esôfago que se abre no cárdia (E), piloro duodenal (P) porção glandular $(\mathrm{G})$, curvatura menor $(\mathrm{Cm})$, curvatura maior $(\mathrm{Cn})$ e fundo $(\mathrm{F})$. D: porção pilórica $(\mathrm{P})$, porção glandular $(\mathrm{G})$, cárdia (Seta fina), prega gástrica (seta vazada), porção aglandular (A) e porção fundica (F). Escala de barra: 1mm. E: ânus (seta) situado na cavidade cloacal (C). Escala de barra: $1 \mathrm{~mm}$.
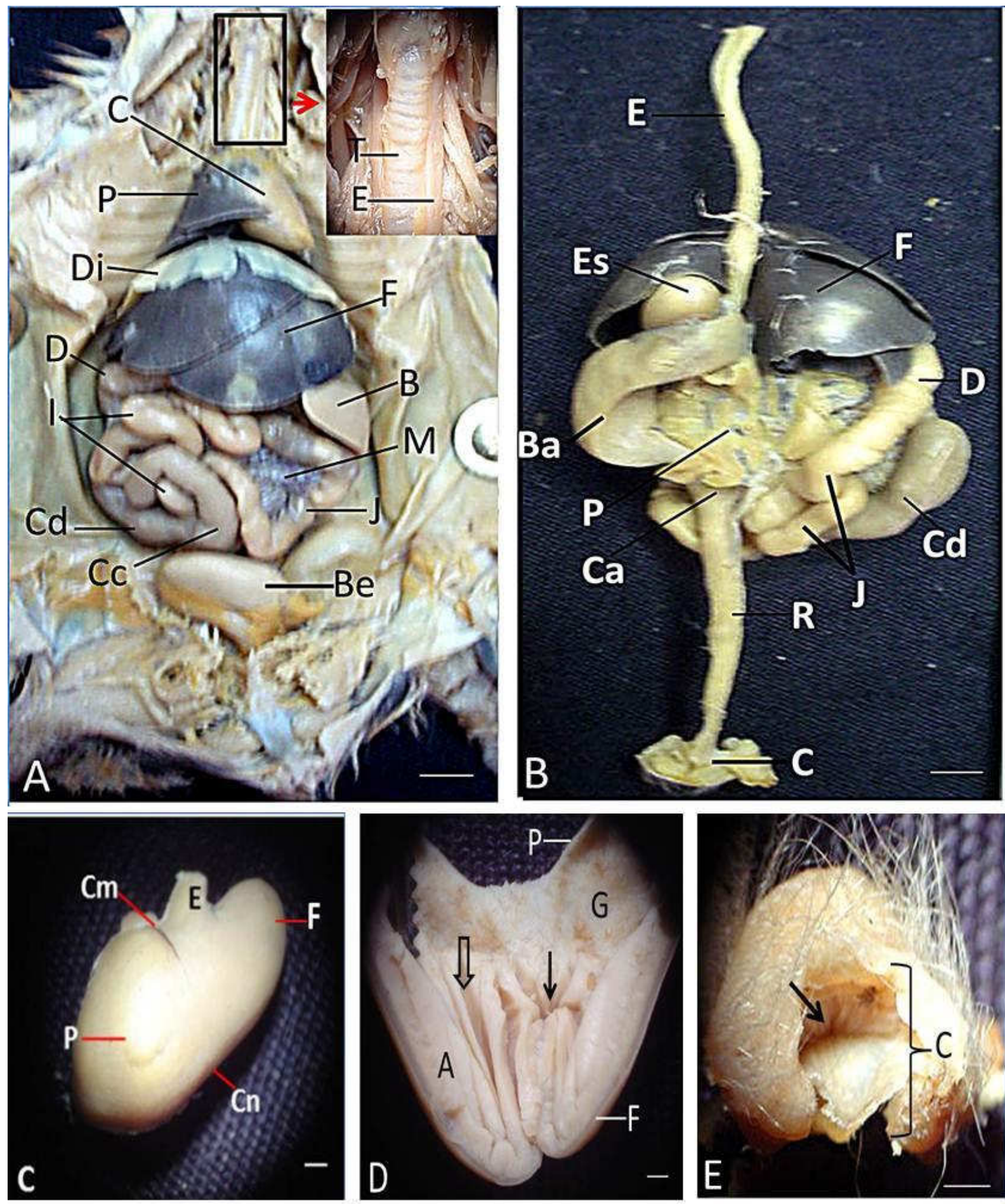
FIGURA 3: Fotomacrografia "in situ" das glândulas salivares, pâncreas e fígado do Gracilinanus microtarsus. A: vista lateral esquerda mostrando as glândulas salivares: mandibular $(\mathrm{Gm})$, sublingual $(\mathrm{Sb})$ e parótida $(\mathrm{Gp})$, base do pavilhão auricular. Escala de barra: $1 \mathrm{~mm}$. B: glândulas salivares mandibulares $(\mathrm{Gm})$; sublinguais $(\mathrm{Sb})$ e parótidas $(\mathrm{Gp})$ em vista ventral. Escala de barra: $1 \mathrm{~mm}$. C: pâncreas (P) e sua relação com o duodeno (D) apresentando aspecto lobulado e formato irregular além de vaso sanguíneo (V), estômago (E) e jejuno (J). Observar a Escala de barra: $1 \mathrm{~mm}$. D: vista ventrolateral do fígado mostrando os lobos: medial direito $(\mathrm{Md})$ e quadrado $(\mathrm{Q})$, os quais estavam paralelos à vesícula biliar (V); lobos lateral esquerdo (Le) e lateral direito $(\mathrm{Ld})$, medial esquerdo $(\mathrm{Me})$ e caudado $(\mathrm{C})$; ducto colédoco $(\mathrm{Cl})$. Escala de barra: $1 \mathrm{~mm}$. E: vista dorsal do fígado mostrando a vesícula biliar ( $\mathrm{Vb}$ ) entre os lobos medial direito $(\mathrm{Md})$ e quadrado $(\mathrm{Q})$; lobos medial esquerdo (Me), lateral esquerdo (Le) e lateral direito (Ld). Escala de barra: $1 \mathrm{~mm}$.
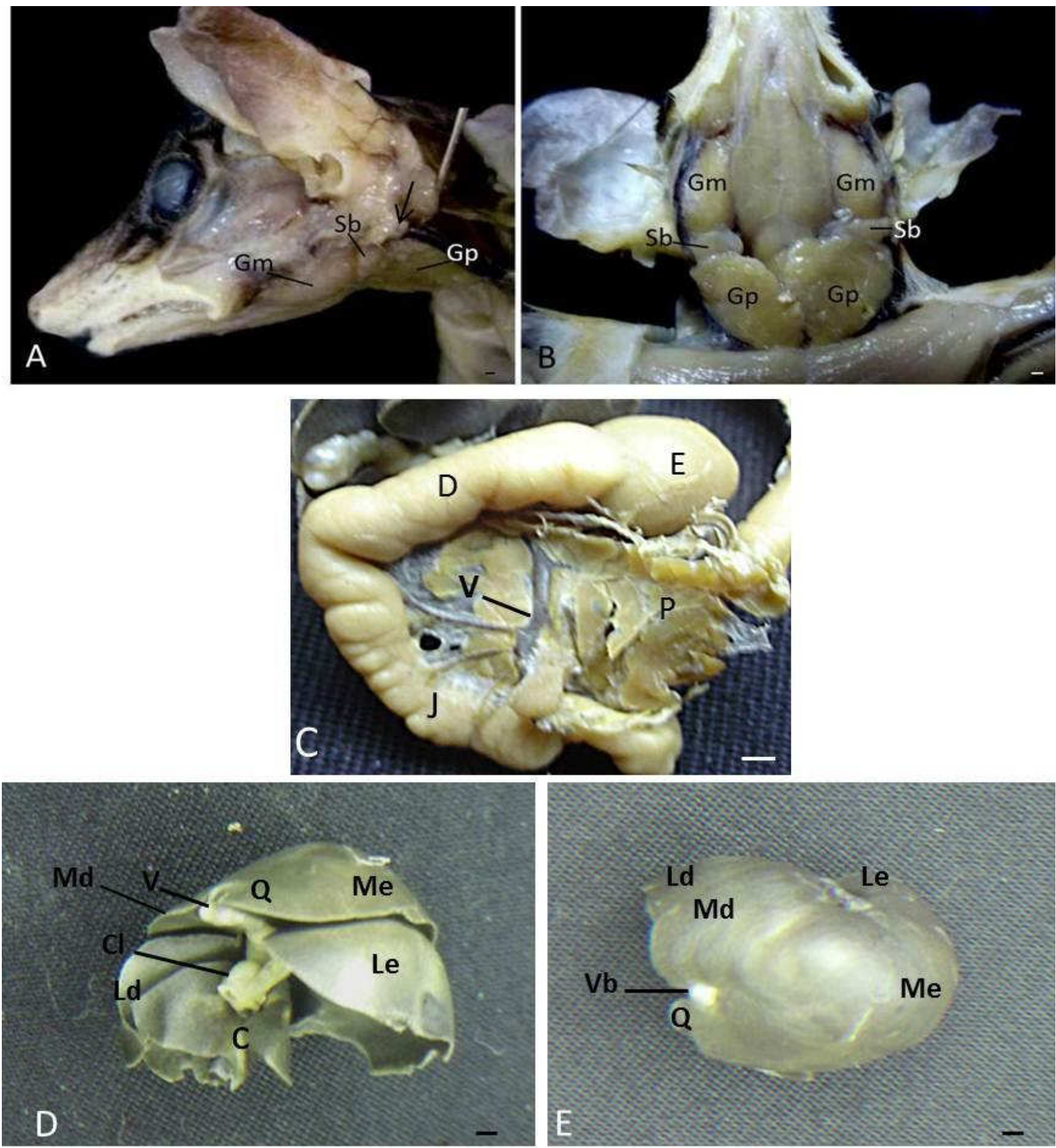


\section{Análise morfométrica}

Os órgãos do aparelho digestório foram mensurados levando em consideração seu formato anatômico e os resultados estão contidos na Tabela 1 .

\section{Discussão}

Todos os animais precisam de matéria prima e energia para seu crescimento, manutenção e reprodução, e a matéria prima utilizada é proveniente dos alimentos. Entre os diversos métodos de ingestão de alimentos, destacam-se: absorção através da superfície corpórea externa; endocitose; ingestão por filtração; ingestão de líquidos e captura de presas (RANDALL et al., 2000).

Neste sentido, os animais desenvolveram um conjunto de órgãos formando um aparelho digestório, o qual foi objeto deste estudo na espécie G. microtarsus, que é responsável pela dispersão de sementes de diversas plantas que fazem parte da sua dieta alimentar, e por esta razão possuem grande importância ecológica, pois a passagem das sementes pelo trato digestório dessa espécie, faz com que as sementes de frutos sofram um processo de quebra de dormência (FONTES et al., 2007), como descrito nos gambás (CÁCERES; MONTEIRO FILHO, 2007; CÁCERES et al., 2008) e morcegos (SATO et al., 2008).

Os órgãos do aparelho digestório encontrados no G. microtarsus têm as mesmas características encontradas nos marsupiais Didelphis albiventris, $D$. aurita, Metachirus nudicaudatus, Philander frenatus, Lutreolina crassicaudata, Monodelphis sorex e Caluromys lanatus (CÁCERES, 2005), em relação aos mamíferos domésticos (DYCE et al., 2010) e roedores como a Chinchilla laniger (CASTRO et al., 2010), a diferença está na ausência de uma cloaca.

O palato duro é cerca de três vezes maior em relação ao palato mole, apresentando o total de oito pregas palatinas e o arco orofaríngeo localizado atrás da língua.

A fórmula dentária dos G. microtarsus é a mesma observada nos Didelphis sp. (CARVALHO et al., 2004), sendo que, as duas espécies pertencem à família
Didelphidae (ORR, 1986; FONTES et al., 2007) com os mesmos hábitos alimentares (FONTES et al., 2007). Em geral, os dentes incisivos servem para cortar, os caninos para furar, os pré-molares para dilacerar e os molares para reduzir o alimento em pequenos fragmentos por meio da mastigação (JERNVALL, 2000).

Neste sentido, sabe-se que os dentes são adaptáveis de acordo com os hábitos alimentares, e apresentam variação de detalhes estruturais entre os vertebrados, fazendo com que os dentes sejam importantes para a sistemática (HILDEBRAND, 1995).

A dentinogênese dos marsupiais Didelphis albiventris têm o início por volta da terceira semana pós-natal, com o esmalte que começa a aparecer e será depositado até a décima semana, e a partir desse estágio entra no período de maturação (AZEVEDO; GOLDBERG, 1987).

Os animais aqui estudados possuem dentes braquidontes, conforme o descrito em animais domésticos como cão e gato (BANKS, 1991) e gambás Didelphis marsupialis (CARVALHO et al., 2004) e diferente dos roedores Chinchilla laniger (CASTRO et al., 2010) e Kerodon rupestris (THOMAZ et al., 2006) que possuem dentes hipsodontes, com a fórmula 2 x I 1/1, C 0/0, P 1/1, M 3/3 característica da maioria dos roedores.

Alíngua dos G. microtarsus está posicionada dentro da cavidade oral, sendo que esta posição é a mesma encontrada nos vertebrados terrestres e mamíferos aquáticos (IWASAKI, 2002), com papilas linguais distribuídas irregularmente sobre a superfície dorsal da língua, como narrado nos roedores Cavia porcellus (KOBAYASHI, 1990), em carnívoros silvestres, como o Nasua nasua (SOUZA et al., 2012), nos marsupiais Didelphis marsupialis (MANÇANARES et al., 2012), nos mamíferos domésticos, como Felis catus e Oryctolagus cuniculus (CHAMORRO et al., 1987), e primatas, como Saimiri sciureus (BRANCO et al., 2011), e Callithrix penicillata (BRANCO et al., 2012), além de outros vertebrados como anfíbios, répteis e aves (IWASAKI, 2002), enquanto nessas mesmas espécies acima citadas, nota-se que a superfície ventral da língua não apresenta papilas. 
Na língua de G. microtarsus são encontradas papilas filiformes, papilas fungiformes e três papilas valadas, semelhante às encontradas em Didelphis marsupialis (MANÇANARES et al., 2012) fato que pode estar relacionado a ambos animais pertencerem a mesma família: Didelphidae (ORR, 1986; FONTES et al., 2007), porém, a língua dos animais do presente estudo ainda possuem papilas fungiformes marginais na margem do ápice e não apresentam a lissa lingual que é característica dos animais carnívoros como Nasua nasua (SOUZA et al., 2012).

As diferenças morfológicas nas línguas de diferentes espécies de vertebrados estão relacionadas com diferentes funções, como captura e manipulação de alimentos, auxílio na deglutição, acasalamento, vocalização e paladar que são próprias de cada espécie (KILINC et al., 2010).

O esôfago do G. microtarsus é um tubo relativamente estreito, constituído por três porções, sendo elas: porção cervical, porção torácica e uma porção abdominal relativamente curta, semelhante ao encontrado em mamíferos domésticos (DYCE et al., 2010) e na Chinchilla lanigera (CASTRO et al., 2010). Nos marsupiais Didelphis albiventris, D. aurita, Metachirus nudicaudatus, Philander frenatus, Lutreolina crassicaudata, Monodelphis sorex e Caluromys lanatus, o esôfago varia em tamanho proporcionalmente ao tamanho do indivíduo (CÁCERES, 2005).

O estômago é considerado a parte dilatada do trato digestório onde começam os processos de digestão nos animais mamíferos como, os animais domésticos (DYCE et al., 2010) e a Chinchilla laniger (CASTRO et al., 2010). Serve como local de armazenamento de alimento, reduzindo o tamanho das partículas alimentares e liberando-as somente quando estão suficientemente quebradas para sofrerem digestão no intestino delgado e grosso (CUNNINGHAM, 2004).

No presente estudo, o estômago monocavitário encontrado no G. microtarsus possui pregas gástricas como descrito nos carnívoros domésticos como cães e gatos (DYCE et al., 2010). O cárdia está localizado cranialmente, entre o fundo e o piloro, como encontrado nos roedores Akodon azarae e Calomys musculinus (ROUAUX et al., 2003).
Cáceres (2005), em seu estudo comparativo com sete espécies diferentes de marsupiais da família Didelphidae, descreve que o estômago sofre variações de acordo com o hábito alimentar da espécie.

Baseado neste estudo, podemos sugerir que o estômago de pequenas dimensões encontrados no $G$. microtarsus, revela que esta espécie possui hábito onívoro, mas preferencialmente insetívoro, de acordo com Pires et al. (2010).

Em espécies de hábito alimentar carnívoro o estômago é grande e elástico para armazenar temporariamente grande quantidade de carne, o contrário do que ocorre em espécies com hábito frugívoro (HILDEBRAND, 1995; CÁCERES, 2005).

$\mathrm{O}$ intestino delgado do guaiquica está dividido em três porções: o duodeno, jejuno e íleo, sendo o jejuno a porção mais longa, conforme descrito em carnívoros domésticos (ELLENPORT, 1986). Além disso, o intestino delgado é muito maior que o intestino grosso, como também encontrado nos marsupiais onívoros Didelphis albiventris, D. aurita, Metachirus nudicaudatus, Philander frenatus, Lutreolina crassicaudata, Monodelphis sorex e Caluromys lanatus, sendo que, espécies com hábito frugívoro como o Caluromys lanatus apresentam um intestino grosso mais desenvolvido que espécies com hábito carnívoro como Didelphis albiventris e D. aurita (CÁCERES, 2005).

No caso dos G. microtarsus, a relação intestino grosso $\mathrm{x}$ intestino delgado parece estar relacionada com espécies com hábitos mais frugívoro que carnívoro (SANTORI et al., 2004; CÁCERES, 2005).

O intestino grosso do G. microtarsus está dividido em três partes: ceco, cólon e reto. O ceco bem desenvolvido nessa espécie pode ter relação com estoque e fermentação de sementes e a absorção de água, vitaminas, eletrólitos e carboidratos como descrito em espécies de hábito insetívoro (CÁCERES, 2005). O cólon também bem desenvolvido pode estar relacionado a absorção de água e eletrólitos, armazenamento do bolo fecal e fermentação da matéria orgânica que escapa da digestão e absorção no intestino delgado (CUNNINGHAM, 2004). 
O reto do animal aqui estudado a exemplo de outros marsupiais está inserido na cloaca e abre-se no ânus. O ânus está situado nesta cavidade comum aos sistemas urogenital e digestório, sendo que, a abertura anal esta situada dorsalmente em relação a abertura genital (CUBAS et al., 2006).

Dentre os órgãos do aparelho digestório do Gracilinanus microtarsus, encontram-se órgãos anexos, como as glândulas salivares, o fígado e o pâncreas, a exemplo do descrito em mamíferos domésticos (DYCE et al., 2010).

Localizamos nesta espécie três glândulas salivares, parótida, mandibular e sublingual. A glândula salivar parótida apresentou-se maior quando comparada às demais (mandibular e sublingual). Estas glândulas possuem posicionamento semelhante ao encontrado nos Didelphis sp. (MARTUCCI et al., 2004), sendo que a glândula parótida possui posicionamento e formato semelhante aos procíonídeos Nasua nasua e Procyon cancrivorus (SANTOS et al., 2012) e a glândula mandibular assemelhou-se ao descrito para Nasua nasua (SANTOS et al., 2010).

Considerada a segunda glândula maior do corpo o pâncreas desempenha tanto a função endócrina produzindo insulina como exócrina (SAMUELSON, 2007). O posicionamento no antímero direito e o formato irregular lobulado e difuso do pâncreas de Gracilinanus microtarsus também é descrito nos Didelphis sp. (CASALS et al. 2004), os quais pertencem à mesma família Didelphidae(ORR, 1986; FONTES et al., 2007). Porém, não se assemelha ao pâncreas com formato em "V" dividido em dois lobos como nos cães e gatos (ELLENPORT, 1986).

O posicionamento do fígado na espécie estudada também é narrado em Didelphis sp. (CASALS et al., 2004), porém, em relação a sua lobação, enquanto o fígado de G. microtarsus é composto por seis lobos, o fígado do Didelphis sp., possui cinco lobos (CASALS et al., 2004), e, neste caso, a proximidade filogenética não garante a similaridade (ORR, 1986; FONTES et al., 2007). Porém, o fígado da Chinchilla lanígera (CASTRO et al., 2010) apresenta lobação semelhante aos nossos achados.
A vesícula biliar situa-se na fossa da vesícula biliar entre as duas partes do lobo medial direito não atingindo a borda ventral do fígado. O ducto colédoco passa para a direita e abre-se no duodeno como nos carnívoros domésticos (ELLENPORT, 1986).

Diante do exposto, conclui-se que nos $G$. microtarsus, os órgãos do aparelho digestório com exceção do ânus que se abre na cloaca como os demais marsupiais, são os mesmos encontrados em animais carnívoros domésticos e roedores. A morfologia da língua e dentes é semelhante ao encontrado nos Didelphis marsupialis. O estômago pouco desenvolvido e o ceco e colón bem desenvolvido são característicos de marsupiais que apresentam hábito predominantemente frugívoro. A morfologia das glândulas salivares e o pâncreas difuso é semelhante ao narrado em Didelphis sp., porém a lobação do fígado difere entre as duas espécies.

Por serem dispersores de semente e por outros estudos mostrarem que as sementes que passasm pelo trato digestório de outros marsupiais sofre quebra de dormência, aumentando o potencial de germinação, o G. microtarsus pode ser utilizado em programas de recuperação de áreas degradadas, com enfoque na dispersão de sementes de plantas pioneiras (CÁCERES; MONTEIRO FILHO, 2007).

\section{Referências}

AZEVEDO, N.; GOLDBERG, M. Post-natal development of tooth structures in Didelphis albiventris. Journal de Biologie Buccale, Paris, v. 15, n. 1, p. 23-35, 1987.

BANKS, W. J. Histologia veterinária aplicada. 2 ed. São Paulo: Manole, 1991. 629 p.

BRANCO, E.; GUIMARÃES, J. P.; MIGLINO, M. A.; LACRETAJR, A. C. C.; ISHIZAKI, M. N.; GOMES, B. D.; MUNIZ, J. A. P. C.; IMBELONI, A.; FIORETTO, E. T.; LIMA, A. R. Ultrastructural aspects of lingual papillae in squirrel monkey (Saimiri sciureus). Microscopy Research and Technique, Malden, v. 74, n. 6, p. 484487, 2011.

BRANCO, E.; PEREIRA, W. L.; DE LIMA, A. R.; FRANCIOLLI, A. L.; RICI, R. E.; MIGLINO, M. A.; MUNIZ, J. A.; IMBELONI, A. Ultrastructural aspects of Callithrix penicillata lingual papillae. Microscopy Research and Technique, Malden, v. 75, n. 3, p. 282284, 2012.

CÁCERES, N. C. Comparative lengths of digestive tracts of seven didelphid marsupials (Mammalia) in relation to diet. Revista Brasileira de Zoologia, Curitiba, v. 22, n. 1, p. 181-185, 2005. 
CÁCERES, N. C.; CASELLA, J.; VARGAS, C. F.; PRATES, L. Z.; TOMBINI, A. A. M.; GOULART, C. S.; LOPES, W. H. Distribuição geográfica de pequenos mamíferos não-voadores nas bacias dos rios Araguaia e Paraná, região Centro-Sul do Brasil. Iheringia, Série Zoologia, Porto Alegre, v. 98, n. 2, p. 173-180, 2008.

CÁCERES, N. C.; MONTEIRO-FILHO, E. L. A. Germination in seed species ingested by opossums: implications for seed dispersal and forest conservation. Brazilian Archives of Biology and Technology, Curitiba, v. 50, n. 6, p. 921-928, 2007.

CARVALHO, A. F.; MANÇANARES, C. F.; CASALS, J.; THOMAZ, J. M.; BELlATINE, T.; MIGLINO, M. A.; AMBRÓSIO, C. E.; ESTEVES, A.; ROQUETO, M. A.; ROSA, R. A. Análise macro e microscópica do dente do gambá (Didelphis sp.). In: ENCONTRO DE PRODUÇÃO ACADÊMICA, 5, 2004, São João da Boa Vista. Anais... São João da Boa Vista: Centro Universitário da Fundação de Ensino Octávio Bastos, 2004. p. 155158.

CASALS, J.; BELLATINE, T.; AMBRÓSIO, C. E.; CARVALHO, A. F.; MARTINS, J. F. P.; ROQUETO, M. A.; MANÇANARES, C. A. F.; MIGLINO, M. A.; HOFFMAN, R. Análise macro e microscópica do pâncreas e fígado de gambá (Didelphis sp.). In: ENCONTRO DE PRODUÇÃO ACADÊMICA, 5, 2004, São João da Boa Vista. Anais... São João da Boa Vista: Centro Universitário da Fundação de Ensino Octávio Bastos, 2004. p. 137-140.

CASTRO, T. F.; DUMMER, R. J.; RICKES, E. M.; PEREIRA, M. A. M. Aspectos morfológicos, morfométricos e topográficos do aparelho digestório de Chinchilla lanigera. Brazilian Journal of Veterinary Research and Animal Science, São Paulo, v. 47, n. 1, p. 86-94, 2010.

CHAMORRO, C. A.; SANDOVAL, J.; FERNANDEZ, J. G.; FERNANDEZ, M.; DE PAZ, P. Estudio comparado de las papilas linguales del Gato (Felis catus) y del Conejo (Oryctolagus cuniculus) mediante el microscópio electronico de barrido. Anatomia, Histologia, Embryologia, Berlin, v. 16, n. 1, p. 37-47, 1987.

COSTA, L. P.; LEITE, Y. L. R.; PATTON, J. L. Phylogeography and systematic notes on two species of gracile mouse opossums, genus Gracilinanus (Marsupialia: Didelphidae) from Brazil. Proceedings of the Biological Society of Washington, Washington, v. 116, n. 2 , 275-292, 2003.

CUBAS, Z. S.; SILVA, J. C. R.; CATÃO-DIAS, J. L. Tratado de animais selvagens: Medicina Veterinária. São Paulo: Roca, 2006. $1354 \mathrm{p}$.

CUNNINGHAM, J. G. Tratado de Fisiologia Veterinária. 3 ed. Rio de Janeiro: Guanabara Koogan, 2004. 579 p.

DYCE, K. M.; SACK, W. O.; WENSING, C. J. G. Tratado de Anatomia Veterinária. 4 ed. Rio de Janeiro: Guanabara Koogan, 2010. 834 p.

EISENBERG, J. F.; REDFORD, K. H. Mammals of the Neotropics: The Central Neotropics. Vol. 3. Chicago: University of Chicago Press, 2000. 610 p.

ELLENPORT, C. R. Sistema Digestivo. In: GETTY, R. (Ed.). Anatomia dos animais domésticos. Vol. 2. 5 ed. Rio de Janeiro: Interamericana, 1981. p. 1445-1464.

FONTES, S. V.; PASSAMANI, M.; JACINTO, C. H.; PEREIRA, M. S.; SANT'ANA, A. P. P. Área de vida e deslocamento de Akodon montensis e Gracilinanus microtarsus em um fragmento no sul de
Minas Gerais. In: CONGRESSO DE ECOLOGIA DO BRASIL, 8, 2007, Caxambu. Anais... Caxambu: SBE, 2007. Versão eletrônica. GONÇALVES, N. N.; MANÇANARES, C. A. F.; MIGLINO, M. A.; SAMOTO, V. Y.; MARTINIS, D. S.; AMBROSIO, C. E.; FERRAZ, R. H. S.; CARVALHO, A. F. Aspectos morfológicos dos órgãos genitais femininos do gambá (Didelphis sp.), Brazilian Journal of Veterinary Research and Animal Science, São Paulo, v. 46, n. 4, p. 332-338, 2009.

HERSHKOVITZ P. The South American gracile mouse opossums, genus Gracilinanus Gardner and Creighton, 1989 (Marmosidae, Marsupialia): a taxonomic review with notes on general morphology and relationships. In: HERSHKOVITZ P. (Ed.). Fieldiana, Zoology: New Series. Vol. 70. Chicago: Field Museum of Natural History, 1992. p. 47-49.

HILDEBRAND. M. Análise da estrutura dos Vertebrados. São Paulo: Atheneu, 1995, $700 \mathrm{p}$.

INTERNATIONAL COMITEE ON VETERINARY GROSS ANATOMICAL NOMENCLATURE. Nomina Anatomica Veterinaria. 5 ed. Hannover: International Committee on Veterinary Embryological Nomenclature ICVEN, 2012. 165 p.

IWASAKI, S-I. Evolution of the structure and function of the vertebrate tongue. Journal of Anatomy, London, v. 201, n. 1, p. 1-13, 2002.

JERNVALL, J. Linking devolopment with generation of noveltry in mammalian teeth. Proceedings of the National Academy of Sciences of the United States of America, Washington, v. 97, n. 5, p. 2641-2645, 2000.

KILINC, M.; ERdoGAN, S.; KeTANI, S.; KeTANI, M. A. Morphological study by scanning electron microscopy of the lingual papillae in the middle east blind mole rat (Spalax ehrenbergi, Nehring, 1898). Anatomia, Histologia, Embryologia, Berlin, v. 39, n. 6, p. 509-515, 2010.

KOBAYASHI, K. Three-dimensional architecture of the connective tissue core of the lingual papillae in the guinea pig. Anatomy and Embriology, Berlin, v. 182, n. 3, p. 205-213, 1990.

MANÇANARES, C. F.; SANTOS, A. C.; PIEMONTE, M. V.; VASCONCELOS, B. G.; CARVALHO, A. F.; MIGLINO, M. A.; AMBROSIO, C. E.; ASSIS NETO, A. C. Macroscopic and microscopic analysis of the tongue of the common opossum (Didelphis marsupialis). Microscopy Research and Techniques, Malden, v. 75, n. 10, p. 1329-1333, 2012.

MARTINS, E. G.; ARAÚJO, M. S. Sex and season affect individuallevel diet variation in the Neotropical Marsupial Gracilinanus microtarsus (Didelphidae). Biotropica, Washington, v. 40, n. 1, p. 132-135, 2008.

MARTUCCI, M.; MALEK, C.; AMBRÓSIO, C. E.; CARVALHO, A. F.; MIGLINO, M. A. Caracterização das glândulas salivares maiores do gamba (Didelphis sp.). In: ENCONTRO DE PRODUÇÃO ACADÊMICA, 5, 2004, São João da Boa Vista. Anais... São João da Boa Vista: Centro Universitário da Fundação de Ensino Octávio Bastos, 2004. p. 146 -150.

ORR, R. T. Biologia dos Vertebrados. 5 ed. São Paulo: Roca. 1986. 5008 p.

PIRES, M. M.; MARTINS, E. G.; SILVA, M. N. F.; REIS, S. F. Gracilinanus microtarsus (Didelphimorphia: Didelphidae). Mammalian Species, Washington, v. 42, n. 851. p 33-40, 2010.

RANDALL, D.; BURGREN, W.; FRENCH, K. Fisiologia animal: 
mecanismos e adaptações. 4 ed. Rio de Janeiro: Guanabara Koogan, 2000. 729 p.

ROUAUX, R.; GIAI, C.; FERNÁNDEZ, N.; BIANCO, V.; De SANTIS, L. J. M. Estructura del estómago em Akodon azarae y Calomys musculinus (Rodentia; Murudae). Mastozoologia Neotropical, Mendoza, v. 10, n. 1, p. 115-121, 2003.

SAMUELSON, D. A. Tratado de Histologia Veterinária. Rio de Janeiro: Elsevier, 2007. 527 p.

SANTORI, R. T.; ASTUA DE MORAIS, D.; CERQUEIRA, E. Comparative gross morphology of the digestive tract in ten Didelphidae marsupial species. Mammalia, Paris, v. 68, n. 1, p.2736, 2004.

SANTOS, A. C.; BERTASSOLI, B. M.; OLIVEIRA, F, D.; OLIVEIRA, D, M.; OLIVEIRA, V. C.; VASCONCELOS, B. G.; CARVALHO, A. F.; MANÇANARES, C. A. F.; ASSIS NETO, A. C. Estrutura macro e microscópica das glândulas salivares parótidas em duas espécies de procionídeos: mão-pelada (Procyon cancrivorus, G. Cuvier, 1798) e quati (Nasua nasua, Linnaeus, 1766). Biotemas, Florianópolis, v. 25, n. 1, p. 93-101, 2012.
SANTOS, A. C.; BERTASSOLI, B. M.; OLIVEIRA, V. C.; ROSA. R. A.; CARVALHO. A. F.; MANÇANARES, C. A. F. Caracterização morfológica das glândulas salivares mandibulares dos quatis (Nasua nasua, Linnaeus, 1758). Revista da Faculdade de Zootecnia, Veterinária e Agronomia, Uruguaiana, v. 17, n. 2, p. 276-286, 2010.

SATO, T. M.; PASSOS, F. C.; NOGUEIRA, A. C. Frugivoria de morcegos (Mammalia, Chiroptera) em Cecropia pachystachya (Urticaceae) e seus efeitos na germinação das sementes. Papéis Avulsos de Zoologia, São Paulo, v. 48, n. 3, p. 19-26, 2008.

SOUZA, A. F.; OLIVEIRA, V. C.; SANTOS, A. C.; ROSA, R. A. CARVALHO, A. F.; MANÇANARES, C. F. Morfologia macro e microscópica das papilas linguais do quati. Pesquisa Veterinária Brasileira, Seropédica, v. 32, n. 3, p. 271-277, 2012.

THOMAZ, J. M.; CARVALHO, A. F.; MIGLINO, M. A.; MANÇANARES, C. A. F.; AMBRÓSIO, C. E.; OLIVEIRA, M. F. Caracterização morfológica dos dentes de mocó Kerodon rupestres: Mammalia: Rodentia. Brazilian Journal of Veterinary Research and Animal Science, São Paulo, v. 43, n. 5, p. 702-707, 2006.

Cidade- Brasil. Disponível em <http:/www.cidade-brasil.com.br/ municipio-alta-floresta.html>. Acesso em: 10 out. 2013. 